\title{
TRANSMISIÓN DEL TRABAJO REPRODUCTIVO FAMILIAR HACIA LAS MUJERES MIGRANTES. VULNERABILIDAD, VIOLENCIA E INVISIBILIDAD DENTRO DEL TRABAJO DOMÉSTICO
}

\author{
TRANSMISSION OF FAMILY REPRODUCTIVE WORK \\ TO MIGRANT WOMEN. VULNERABILITY, VIOLENCE \\ AND INVISIBILITY IN DOMESTIC WORK
}

\author{
ROCÍO CÁRDENAS-RODRÍGUEZ* \\ FABIOLA ORTEGA-DE-MORA**
}

\begin{abstract}
RESUMEN
La creciente incorporación de las mujeres migrantes en el mercado laboral, y en concreto en el sector de los cuidados, justifica la necesidad de analizar las repercusiones de la transmisión del trabajo reproductivo familiar hacia las mujeres migrantes, estudiando la vulneración de los derechos que se produce antes y durante su incorporación al mercado laboral. Para ello, se ha realizado una revisión bibliográfica de los últimos avances en la materia, utilizando como descriptores "migrantes", "mujer", "cadena de cuidados", entre otros. El presente artículo demuestra, a través de los últimos datos existentes, la invisibilidad, exclusión y discriminación que sufren las mujeres migrantes en la Cadena Global de los Cuidados.
\end{abstract}

PALABRAS CLAVE: mujeres, migrantes, cadena global de los cuidados, trabajo doméstico, vulnerabilidad.

\begin{abstract}
The increasing incorporation of migrant women in the labor market and, specifically, in the care sector, justifies the need to analyze the repercussions of the transmission of family reproductive work to migrant women, studying the violation of rights that occur before and during its incorporation into the labor market. For this, a bibliographic review of the latest advances in the subject has been carried out, using as descriptors "migrants", "women", "care chain", among others. This article demonstrates, through the latest existing data, the invisibility, exclusion and discrimination suffered by migrant women in the Global Care Chain.
\end{abstract}

KEYWORDS: women, migrants, global care chain, domestic work, vulnerability.

* Doctora en Ciencias de la Educación. Facultad de Ciencias Sociales. Universidad Pablo de Olavide. Dpto. de Educación y Psicología Social. Edificio 11. Ctra. de Utrera, km. 1. 41013. Sevilla. España. mrcarrod@upo.es

** Máster en Criminología y Ciencias Forenses. Facultad Ciencias Sociales. Universidad Pablo de Olavide. Ctra. de Utrera, km. 141013. Sevilla, España. faortde@upo.es 


\section{INTRODUCCIÓN}

A pesar de las diferentes medidas legales y políticas que se han promovido a nivel nacional e internacional para asegurar el reparto igualitario en el trabajo reproductivo, no se ha conseguido establecer unas medidas eficaces que aseguren dicha igualdad. Entendemos por trabajo reproductivo, el "trabajo destinado a satisfacer las necesidades de la familia" (Larrañaga, Arregi \& Arpal, 2004). Tradicionalmente, el modelo de organización social que ha existido, ha promovido una división sexual del trabajo en la que los hombres se encargaban de la labor productiva, vinculado con el ámbito público, y las mujeres, con el trabajo doméstico y de cuidado, relacionado con el espacio privado.

No obstante, a finales del siglo XX, concretamente en los años 70, con el incremento del número de mujeres que acceden al mundo laboral remunerado, empieza a producirse una modificación en el sistema tradicional (Durán, 1972; Maruani, 2000; Rodríguez y Larrañaga, 2002-2004; Alcañiz, 2015). Las mujeres comienzan a adquirir un doble rol: el de cuidadora y el de trabajadora, siendo difícil conciliar la vida laboral con la familiar. Ante esta realidad, se han implementado algunas políticas de empleo orientadas a favorecer dicha conciliación (Alcañiz, 2015); no obstante, las medidas públicas adoptadas no han sido suficien- tes, dando lugar a la denominada Crisis de Cuidados.

Ante el debilitamiento del Estado de Bienestar y el aumento de personas que necesitan cuidados (Legarreta, 2017), las familias han tenido que plantearse algunas alternativas como "externalizar parte de las tareas que antes se realizaban en los hogares, o salarizar parte del trabajo de cuidados en lo doméstico" (Orozco, 2007, p.5). En dicha externalización adquieren un rol principal las mujeres migrantes, al ser ellas las encargadas de realizar el trabajo doméstico que antiguamente desempañaban las mujeres en su propio hogar.

La transmisión del trabajo reproductivo familiar hacia las mujeres migrantes ha ido incrementándose considerablemente en los últimos tiempos. No obstante, las condiciones de este tipo de empleo suelen ser precarias y normalmente no están bien remuneradas (Orozco, 2007). Las diversas investigaciones muestran cómo las mujeres que deciden iniciar un proceso migratorio se enfrentan a una situación compleja, corriendo un gran riesgo debido a la mayor vulnerabilidad que presentan durante todo el recorrido (Asakura \& Torres, 2013), tanto en aspectos relacionados con el ciclo de la vida, como con factores socio-económicos (Montoro-Gurich, 2017, p.449).

Desde antes de iniciar el proceso migratorio, las mujeres deben afrontar ciertos pre- 
juicios sociales. Debido al patriarcado que sigue imperando en nuestro ordenamiento social, ellas son las encargadas del ámbito reproductivo, teniendo que proporcionar cuidados a toda la familia (Herrera, 2012) y en especial a los hijos e hijas. Esta concepción condiciona el proceso migratorio de las mujeres antes de iniciar su partida, pues deben hacer frente a una gran presión psicológica, entre otros motivos, por no asumir los estereotipos que socialmente les son impuestos (Parella \& Speroni, 2018), relacionados con el cuidado y la protección. La mujer con carga familiar que decide migrar, suele presentar un gran sentimiento de culpa por dejar a sus hijos e hijas en su país (Ciurlo, 2015).

Por otro lado, una vez que inician el recorrido, deben hacer frente a más obstáculos que los hombres por el simple hecho de ser mujeres. Durante todo el camino están expuestas a una continua violencia. En muchas ocasiones se produce una cosificación de la mujer (Asakura \& Torres, 2013). Cuando viajan en grupo, los hombres acuerdan utilizar el cuerpo de la mujer como medio de pago para poder cruzar la frontera (Álvarez, 2010 citado por Asakura \& Torres, 2013). Por ello muchas mujeres, antes de comenzar el proceso, adoptan medidas de protección como inyecciones anticonceptivas o dispositivos intrauterinos, evitando tener embarazos no deseados.
No obstante, dicha violencia no finaliza cuando llegan al país de destino, ya que siguen estando sometidas a situaciones adversas que dificultan su estancia. Muchas se ven abocadas a un tipo de violencia más sutil. Ejemplo de ello sería la posición que adquieren en el mercado laboral en el que las mujeres se ven sometidas a una triple discriminación: por cuestión de género, de etnia/raza y de economía o clase (Juliano 2000; Solé 2000; Parella 2003; Trujillo \& Almeda, 2017).

Muchas de ellas sufren una gran explotación laboral y discriminación en un mercado de trabajo significativamente segmentado, en el que se han producido ejes nuevos de desigualdades (Ezquerra, 2010). Antiguamente, en el mercado laboral existía una gran diferencia entre hombres y mujeres, pero con la incorporación de las mujeres migrantes, existe un nuevo factor diferenciador entre las mujeres: el país de origen, siendo este uno de los tres ejes claves que marcan la posición de subordinación de las migrantes: origen nacional, posición socioeconómica y situación administrativa (Ezquerra, 2012).

En el país de destino, las mujeres suelen desarrollar diferentes tipos de trabajo no cualificados, a pesar de la formación que puedan tener, ya que no tienen muchas alternativas para elegir, accediendo siempre a un mercado feminizado y devaluado socialmente (Castelló, 2009). 
Algunas de las mujeres desarrollan tareas en la agricultura, otras consiguen recursos económicos gracias a actividades sexuales y muchas de ellas se dedican a los cuidados. Hoy en día, gran parte del trabajo reproductivo familiar se ha transmitido a las mujeres migrantes, transfiriéndose unos ejes de poder entre los que podemos señalar: el género, la etnia, la clase social y el lugar de procedencia (Pérez, Paiewonsky y García, 2008, Monreal, Cárdenas \& Martínez, 2019). Esto genera una mayor desigualdad y subordinación de la mujer migrante, ya que esta condición influye de manera determinante en su "acceso a derechos y oportunidades así como a las situaciones de privilegio o de exclusión que se derivan" (Anthias, 1998 citado por Oso \& Parella, 2012, p.14).

En el presente artículo realizaremos una revisión bibliográfica del rol que adquiere la mujer migrante en el trabajo doméstico internacional, analizando la violencia y precariedad a la que son sometidas y el estado actual a nivel internacional.

\section{DATOS ESTADÍSTICOS}

\section{DE LA EXTERNALIZACIÓN}

DEL TRABAJO REPRODUCTIVO. MUJERES MIGRANTES AL CUIDADO

\section{FAMILIAR}

Según la Organización Internacional del Trabajo (2018b), en el año 2017 hubo aproximadamente 258 millones de personas que migraron hacia otro país. De ellos, 164 millones fueron por cuestiones laborales. Esto indica que el 59,2 \% del total de la población que migró durante el 2017 fue una migración laboral, la cual es entendida como "movimiento de personas del Estado de origen a otro con un fin laboral" (OIM, 2006, p.40).

Las migraciones laborales tradicionalmente la han realizado los varones debido a la sociedad patriarcal que ha imperado a lo largo de la historia, donde el hombre debía cumplir su rol productivo. No obstante, esa visión ha ido evolucionando a lo largo del tiempo.

Aunque aún son muchas las migraciones laborales realizadas por varones, el porcentaje de mujeres que migran en busca de un trabajo ha aumentado. En el año 2017, de los 164 millones de personas que migraron para trabajar, 95'7 millones eran hombres y 68'3 millones eran mujeres, lo que supone un $58,4 \%$ y un 41,6 \% respectivamente (Organización Internacional del Trabajo, 2018b). Si bien sigue persistiendo una mayor proporción de varones, las cifras han comenzado a equipararse.

En los últimos tiempos se han planteado diversas explicaciones que justifican la mayor representatividad del hombre en los movimientos migratorios internacionales por cuestiones laborales. Entre ellas, podemos destacar las dificultades que tienen las mujeres para conseguir empleo debido a la vulnerabilidad y discriminación que siguen sufriendo. Junto a ello, la Organización Internacional del Trabajo, también señala: la estigmatiza- 
ción social; las políticas y leyes discriminatorias y la violencia/acoso que deben enfrentar por el simple hecho de ser mujeres, tal y como se ha explicado en los apartados anteriores. Estos aspectos influyen también en el salario y las condiciones precarias a las que se encuentran sometidas (Organización Internacional del Trabajo, 2018a).

A su vez, dichas cuestiones influyen en el tipo de labor que desempeñan. Normalmente, las personas migrantes suelen desarrollar trabajos relacionados con ciertos sectores económicos. Según los datos proporcionados en la Conferencia Internacional del Trabajo (2017), en relación al año 2013, donde el número de migrantes fue de 106,8 millones, los trabajos que realizaron las personas migrantes estuvieron relacionados con las siguientes ramas económicas: trabajo doméstico (7,7 \%), Agricultura (11,1 \%), Industria, incluida manufactura y construcción (17,8 \%) y otros servicios $(63,4 \%)$.
Dentro de estos sectores no existe una representatividad masculina y femenina igualitaria, siendo significativa la brecha por cuestión de género que existe en algunas de las ramas económicas. Los hombres suelen desempeñar trabajos relacionados con la industria y la agricultura. Por el contrario, las mujeres realizan labores en la agricultura, en la misma proporción que los hombres, y en el sector de los servicios. En dicho sector, las migrantes tienen una representatividad significativamente mayor que la de los hombres, representando el 73,4\% de todos los trabajos domésticos realizados, lo que supone que 8,45 millones de mujeres migrantes se dedican a dicho sector.

Sin embargo, no en todas las regiones existe la misma demanda en el sector de los servicios. A continuación, mostramos una gráfica en la que se refleja la distribución del trabajo doméstico en el año 2013 en función de la región.

Figura 1. Distribución del trabajo doméstico por región (2013)

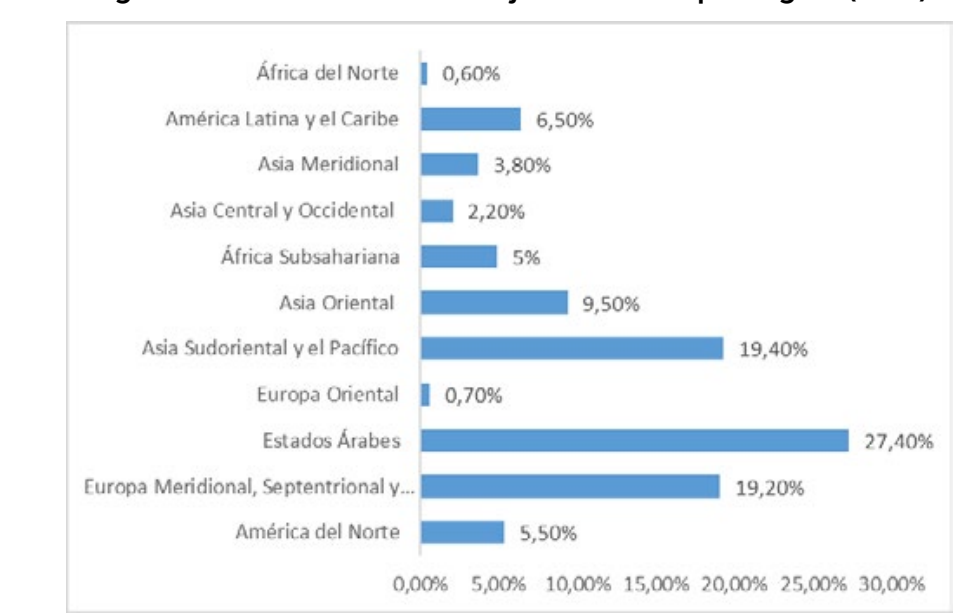

Fuente: Elaboración propia a partir de Organización Internacional del Trabajo (2015). 
Tal y como se observar en la gráfica, la región con mayor número de migrantes dedicados al trabajo doméstico son los Estados Árabes (27,40 \%), seguido por Asia Sudoriental y el Pacífico (19,40 \%) además de Europa Meridional, Septentrional y Occidental (19,20 \%). Sin embargo, en regiones como África del Norte (o,60 \%) y Europa Oriental (o,70 \%), existe una representatividad menor de personas migrantes dedicadas al cuidado doméstico.

Dentro de estas regiones, dependiendo de diversas cuestiones, como por ejemplo aspectos culturales o económicos, la proporción de mujeres y hombres en dicho sector es diferente, tal y como se muestra en la Figura que aparece a continuación.

Figura 2. Distribución del trabajo doméstico de migrantes por sexo

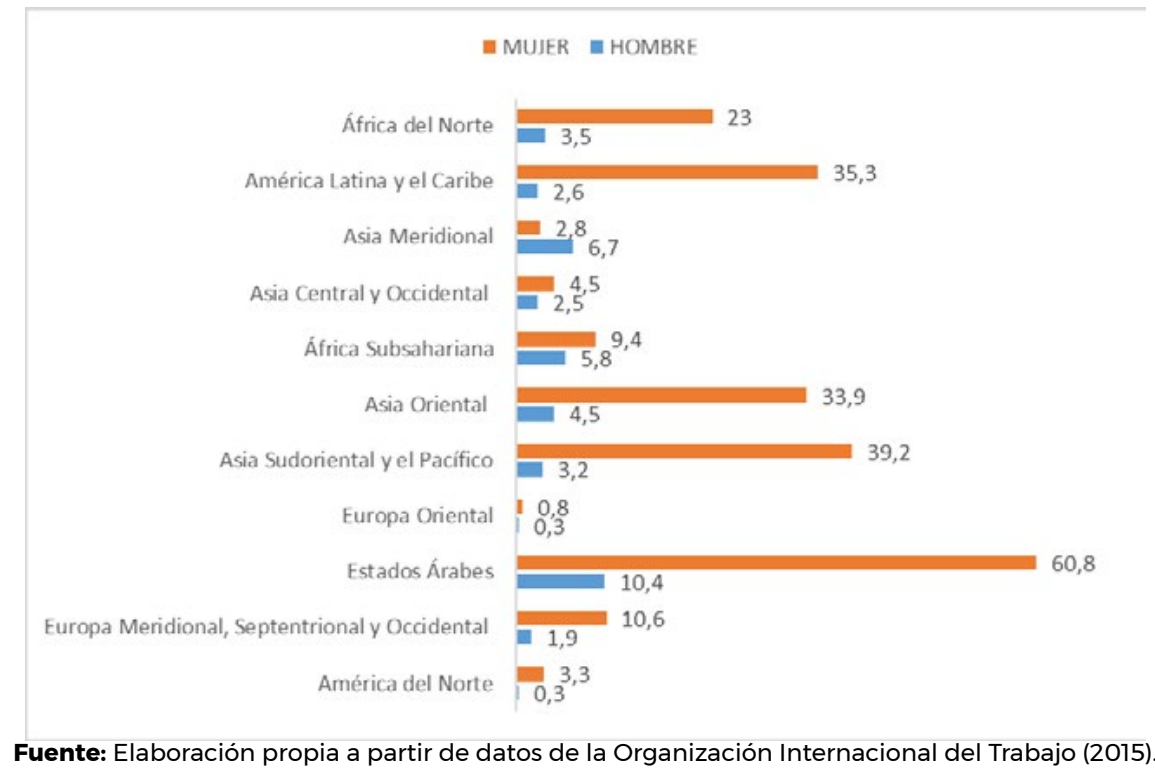

En la Figura se puede observar cómo la mayoría de personas migrantes que se dedican al cuidado doméstico en las diferentes regiones son mujeres. En alguna de ellas, como por ejemplo los Estados Árabes, Asia Sudoriental y el Pacífico o América Latina y el Caribe, la diferencia es significativamente mayor. Por el contrario, solo en Asia Meridional la proporción de hombres que se dedican a dicha labor es mayor que la de mujeres.
Aunque en el 2013, año en el que se realizó el último estudio cuantitativo y específico sobre el trabajo doméstico a nivel global, la diferencia existente entre hombres y mujeres migrantes en el trabajo doméstico era significativo. Se prevé que el número de migrantes dedicados a dicho sector y la diferencia por cuestión de sexo sigan creciendo.

En la sociedad capitalista en la que nos encontramos inmersos, donde los progre- 
sos en todas las áreas del saber, entre ellas la medicina, avanzan de manera vertiginosa, la esperanza de vida de la población ha aumentado significativamente. A nivel mundial, en los últimos años la esperanza de vida media ha pasado de 67 a 70 años, lo que ha supuesto un incremento de tres años (Naciones Unidas, 2019). En los países desarrollados, la población es más longeva, aunque no siempre se encuentran en un estado de salud adecuado, necesitando la ayuda y cuidado de otras personas.

Se prevé que para el año 2025, el número de personas mayores será de 1.200 millones aproximadamente (Hechavarría, Ramírez, García \& García, 2018). Ante esta situación y debido al descenso del número de nacimientos, concretamente en los países desarrollados, aumentará la demanda de cuidados a nivel internacional, adquiriendo una mayor dimensión la Cadena Global de Cuidados que existe en la actualidad.

Si continuamos con el patrón que tradicionalmente ha existido en nuestro ordenamiento social, las mujeres seguirán desarrollando el rol reproductivo que tradicionalmente se le ha impuesto, perpetuando la discriminación por razón de género y el desarrollo de trabajos precarios y mal remunerados. Esto seguirá reproduciendo las desigualdades y vulneraciones de los derechos de las mujeres migrantes.

\section{MUJERES MIGRANTES Y TRABAJO REPRODUCTIVO FAMILIAR. OMISIÓN DEL LUGAR DEL HOMBRE Y VULNERABILIDAD}

Numerosos estudios, como los realizados por la International Labour Organization (2015, 2018b), se han centrado en el análisis de la economía familiar y el trabajo reproductivo del hogar, así como las familias trasnacionales y la importancia de la cadena de cuidados para el mantenimiento del hogar; sin embargo, aún existen pocas investigaciones que centren su atención en el análisis de la cadena de cuidados desde una perspectiva crítica de análisis de género.

Según Molano et al. (2012), las mujeres migrantes se suelen insertar laboralmente en el sector del hogar. Por ejemplo, en Argentina el $69 \%$ de este sector es ocupado por mujeres peruanas, y el 58,1\% por mujeres paraguayas; y en España, el $51,8 \%$ está desempeñado por bolivianas y el $56,2 \%$ por paraguayas. Es decir, el empleo del hogar es un sector altamente feminizado y desempeñado por mujeres migrantes.

En este contexto, las cadenas de cuidado siguen reproduciendo una desigualdad social y una fuerte desigualdad de género dentro de la organización del cuidado, ya que el trabajo reproductivo sigue quedando en manos de las mujeres, de otras mujeres, y siguen siendo consideradas 
una tarea de escaso valor social y fuertemente estigmatizada. Gonzálvez (2010) plantea que la maternidad, el cuidado y la crianza de la familia suelen determinar la posición social de las mujeres migrantes y no migrantes, y condicionan sus proyectos personales, familiares y migratorios. De hecho, existe una corriente en determinados estudios (Hochschild, 2000; Bettio et al., 2006) que analizan la migración femenina como un fenómeno a través del cual las sociedades más ricas "compran" el cuidado y el afecto de las mujeres migrantes más pobres, a bajo coste. Desde hace algunas décadas las migrantes procedentes de países con menos recursos suelen ser las encargadas de realizar el trabajo reproductivo de mujeres y hombres de los países con más recursos, aumentando aún más la desigualdad dentro de las mujeres que tiene más privilegios y las que no tienen recursos ni posibilidad de tenerlos.

En este contexto las mujeres migrantes se incorporan a un mercado laboral vinculado a la reproducción social (cuidados del hogar, de personas) y con condiciones de máxima vulnerabilidad, marginadas, explotadas e invisibles a la sociedad. Esta situación deriva en una desigualdad entre mujeres que desarrollan un trabajo productivo en la sociedad y para ello utilizan a mujeres con menos recursos para que desarrollen el trabajo reproductivo en los hogares, manteniendo la diferenciación de roles de género y transfirien- do el trabajo reproductivo a mujeres con menos recursos, por lo que se mantiene la desigualdad de género en las tareas del hogar y se establece una mayor desigualdad entre las mujeres. De todo ello se evidencia que en la sociedad no se ha producido un verdadero reparto equitativo de las tareas reproductivas en las parejas, sino que se han transferido esas tareas a mujeres con menos recursos y posibilidades. Las mujeres se han incorporado al espacio productivo sin que los hombres lo hagan en el reproductivo, sin producirse un reparto efectivo de las tareas del hogar y cuidado, por lo que recurren a externalizar ese trabajo a través de las mujeres migrantes, produciéndose lo que Aguilar (2010, p.202) denomina "trasvases generizados de la función de cuidado, siempre entre mujeres".

Los estudios de Parella (2003) indican que este sector laboral está marcado por la precariedad, el desprestigio social y los bajos salarios, un sector que han abandonado las mujeres autóctonas con mayor cualificación al insertarse en un sector laboral de mayor prestigio y han recurrido a mano de obra extranjera para cubrir este sector y sus necesidades de cuidado de hogar. En esta misma línea, Aguilar (2010, p.215) sostiene que las que denomina "otras cuidadoras" sufren una triple discriminación como mujeres, trabajadoras y extranjeras ya que desarrollan un empleo de cuasi-servidumbre, estigmatizado socialmente como precario; 
muchas de ellas en situación irregular. Además, sufren la marginación por ser mujeres extranjeras.

Según Aguilar (2010, p.215) para la gran mayoría de inmigrantes las oportunidades laborales se presentan sólo en aquellos empleos menos deseados por los nacionales, porque están peor pagados, son duros, sucios, inestables, estacionales, inseguros, etc.

Por ello, las mujeres inmigrantes quedan relegadas a los empleos que se consideran específicamente femeninos por la sociedad externalizando las tareas de cuidado y del hogar.

A su vez, este es un sector laboral invisibilizado; desarrollan tareas que se realizan en el interior del hogar, tanto las domésticas como el cuidado de ancianos/as y menores, lo que repercute en la invisibilización de su trabajo como cuidadoras y en su escasa valoración, además de una mayor vulnerabilidad al quedar todo el trabajo bajo la privacidad del hogar (Martínez, 2010). Esta invisibilidad contribuye a que las tareas del cuidado y del hogar sean un trabajo que en la sociedad no es reconocido como productivo, por lo tanto, carece de valor de cambio para la sociedad.

Otro elemento que potencia la vulnerabilidad de estas mujeres es la situación administrativa en la que se encuentran. Las mujeres migrantes en situación irregular suelen incorporarse a este sector laboral a través de redes, de familiares y amigos que contactan con los hogares empleadores, pudiendo los empleadores incumplir las condiciones laborales de salario, horario, etc., al no tener la empleada posibilidad de reclamar vacaciones, cumplimiento de horarios, horas extras, antigüedad, etc. Todo esto sitúa a las mujeres migrantes cuidadoras en condiciones de vulnerabilidad ante la explotación laboral sin poder de negociación para mejorar sus condiciones laborales. Según Aguilar (2010), los sueldos en este sector para las mujeres migrantes son muy bajos (la mitad cobra menos del Salario Mínimo Interprofesional), y muchas de ellas tienen que pagar vivienda, alimentación, Seguridad Social (un tercio declara pagar ella misma de todas las cuotas de la Seguridad Social, incluyendo las que corresponderían al empleador), y en algunos casos, deben pagar la deuda que contrajeron para poder realizar su proyecto migratorio.

A esto se les suma que la jornada laboral suele durar más de las 40 horas a la semana, y que no cobran horas extras ni vacaciones. Por todo ello, podemos afirmar que esa situación está favoreciendo una violencia hacia las mujeres extranjeras insertadas en un ámbito laboral precario y muy estigmatizado, una violencia psicológica, económica, estructural, y en algunos casos física.

Según Aguilar (2010, 219),

las mujeres inmigrantes están expuestas a diferentes formas de vio- 
lencia, entre ellas la violencia estructural, que son el resultado de los procesos de estratificación social. Se trata de mecanismos cuya consecuencia es que el acceso, reparto o posibilidad de uso de los recursos, es resuelto sistemáticamente a favor de la población autóctona.

\section{REFLEXIONES FINALES}

La transmisión del trabajo reproductivo familiar hacia las mujeres migrantes ha tenido un gran impacto a nivel internacional. Si bien las familias que demandan cuidados, obtienen un gran beneficio de la labor realizada por las mujeres procedentes de otros países, para las migrantes la realidad es más compleja.

La incorporación de las mujeres migrantes al mercado laboral, concretamente al sector de los servicios y del cuidado doméstico, supone una perpetuación del rol reproductivo que tradicionalmente se le ha asignado. Esta incorporación a un trabajo poco calificado supone, en muchas ocasiones, una vulneración de los principios y derechos fundamentales en el ámbito laboral, lo que se denomina, junto a otras cuestiones, "costos de la migración laboral" (Conferencia Internacional del Trabajo, 2017, p.19).

En el caso de las mujeres, el "costo de la migración laboral” suele ser mayor que el de los hombres. Uno de los motivos principales se debe al sector laboral en el que se insertan. El trabajo doméstico tradicionalmente ha sido una tarea que no ha estado remunerada económicamente y que, por lo tanto, no ha tenido un reconocimiento social. Ha sido un trabajo socialmente invisibilizado. Si bien, las mujeres migrantes reciben una remuneración económica por la labor que desempeñan, sigue siendo un trabajo poco valorado por la sociedad, lo que refuerza la invisibilidad de las mujeres migrantes.

Por otro lado, esta segmentación del mercado contribuye a la discriminación que sufren las mujeres migrantes. Tal y como se ha indicado anteriormente, las mujeres sufren una triple discriminación: por razón de género, de etnia/raza y de economía o clase (Juliano, 2000; Solé, 2000; Parella, 2003). Dichas razones son ejes claves que determinan la situación de vulnerabilidad de estas mujeres.

Según estudios que se han realizado recientemente, uno de los grupos más vulnerables son las mujeres migrantes jóvenes que se dedican al trabajo doméstico, ya que se encuentran expuestas a un mayor tipo de violencia física y sexual en su entorno laboral (O'Neil, Fleury \& Foresti, 2016). Estos aspectos nos llevan a considerar tres elementos claves de discriminación: ser mujer, ser migrantes y ser trabajadoras domésticas.

En primer lugar, ser mujer en el ordenamiento social imperante implica una cuestión de inferioridad. Como se ha comentado 
con anterioridad, en el sistema sexo-género tradicional, ser hombre o mujer ha supuesto una división de los roles y de los espacios entre ambas categorías sexuales, diferenciando y excluyendo los distintos ámbitos. En esta división, la mujer siempre ha estado relegada a la figura del varón.

En segundo lugar, ser migrante normalmente supone

un limbo jurídico que, desde la perspectiva de los derechos humanos es una tierra de nadie donde los que se han alejado de su tierra, ciudad, familia y recursos sociales se encuentran en estado de gran vulnerabilidad. (Petit, 2002, p.9)

La situación de las personas migrantes, en general, suele ser muy compleja. En la mayoría de las ocasiones salen de su país de origen en busca de unas mejores condiciones de vida. Esto implica desvincularse, no solo de sus seres queridos, sino también de su cultura y costumbres.

En tercer lugar, ser trabajadora doméstica, como se ha comentado anteriormente, implica insertarse en un sector del mercado laboral significativamente invisibilizado e infravalorado. Es un empleo que no está reconocido socialmente y cuyos derechos laborales están muy difusos, generándose un espacio de exclusión.

Estos ejes contribuyen al estigma y prejuicios a los que se encuentran sometidas las mujeres migrantes. Si bien tradicio- nalmente, debido a la construcción social, han estado sometidas a una mayor estigmatización que los hombres, en el caso de las migrantes esta situación se acentúa. Es evidente que, en la actualidad, existe una significativa diferencia entre las mujeres atendiendo al origen, procedencia y clase social a la que pertenezcan.

Es por ello que, en el servicio de cuidados y trabajo doméstico, se crea un espacio de exclusión independientemente de que la persona que se encuentre a cargo del núcleo familiar sea un hombre o una mujer. Dicho contexto genera unas relaciones de dependencia entre las trabajadoras y las empleadoras/es, especialmente en lo que respecta a las cuestiones legales para la regularización. Estas relaciones, generan una situación de subordinación de la migrante en su vida laboral, la cual afecta de manera directa en lo personal y en lo social.

Muchas de las mujeres que se encuentran desarrollando una labor en el servicio de cuidados o en las tareas domésticas, lo realizan de manera ilegal. Muy pocas son las que tienen una situación regularizada conforme se establece en el Real Decreto 1620/ 2011 que desarrolla el Art. 2.1. del Estatuto de los Trabajadores.

$\mathrm{Al}$ no poder disponer de los derechos que le corresponden, se enfrentan a una situación de mayor vulnerabilidad; no se le reconocen sus derechos y, en ciertas oca- 
siones, se utiliza la situación irregular en la que se encuentran como medio de coacción para que acceda a realizar todas las tareas y condiciones que las empleadoras o empleadores deseen.

Todas estas cuestiones demuestran cómo el género, hoy tiene que considerarse como una categoría analítica relevante en los estudios migratorios, ya que es el eje angular que articula todo el proceso, desde antes de iniciar la partida en el país de origen, hasta su inclusión en el país de destino.

Es evidente que las trabajadoras migrantes tienen más probabilidades que los trabajadores migrantes de experimentar discriminación por motivos de sexo, nacionalidad y su condición de migrantes, entre otros motivos de discriminación prohibidos. (Conferencia Internacional del Trabajo, 2017, p.21)

Si bien, es una realidad compleja y multifactorial, es necesario promover un cambio y fomentar la visibilización de las mujeres migrantes. Las últimas investigaciones realizadas demuestran que la migración laboral, y en concreto la relacionada con trabajos domésticos y prestación de cuidados, aumentará significativamente debido al crecimiento que se prevé de las tasas de dependencia de la vejez durante el período comprendido entre los años 2015 y 2050, especialmente en Europa, América del Norte, América Latina, El
Caribe y Oceanía (Conferencia Internacional del Trabajo, 2017).

Esto demuestra la necesidad de adoptar medidas que permitan mitigar la vulneración y riesgo a las que se encuentran sometidas las mujeres migrantes y para ello es fundamental atender el ámbito laboral y la transmisión del trabajo reproductivo que realizan las familias hacia las migrantes.

Es evidente que existe una crisis de cuidados, que a través de la cadena Global de Cuidados que se ha creado, se ha intentado hacer frente a dicha realidad. No obstante, es necesario que repensemos esta cadena... la repercusión que tiene a nivel global, los perjuicios que supone para muchas de las personas que participan en ella y las medidas que serían convenientes adoptar en los diferentes contextos, no solo en los países de destino, sino también en los de origen y tránsito, proporcionando de este modo una mayor seguridad y menor vulnerabilidad de las mujeres migrantes.

Como bien plantean Bastia \& Busse (2011), Pedone, Agrela \& Gil (2012), Sassen (1999), y Fuentes \& Agrela (2016), la Cadena Global de Cuidados debe ser considerada como una oportunidad de las mujeres migrantes para insertarse en el mercado laboral y obtener los recursos económicos necesarios para favorecen su autonomía personal. Es necesario analizar la repercusión de dicha realidad 
y cuestionar de manera crítica la situación de vulnerabilidad, estigmatización e invisibilidad que supone dicho proceso migratorio.

\section{REFERENCIAS BIBLIOGRÁFICAS}

Aguilar, M. J. (2010). «Las otras» cuidadoras: mujeres inmigrantes en el servicio doméstico y trasvases generizados en el ámbito territorial del bienestar. Alternativas, 17, 201-220.

Alcañiz, M. (2015). Género con clase: la conciliación desigual de la vida laboral y familiar. RES, (23), 29-55.

Álvarez, S. (2010). Frontera sur chiapaneca: el muro humano de la violencia. Análisis de la normalización de la violencia hacia la migración indocumentada en tránsito en el espacio fronterizo Tecún Umán-Ciudad Hidalgo-Tapachula_HuixtlaArriaga. Tesis de maestría en Antropología Social en la Universidad iberoamericana, México.

Anthias, F. (1998). Rethinking social divisions: some notes towards a theoretical framework. Sociological Review, 46(3), 505-53.

Asakura, H., \& Torres, M. (2013). Migración femenina centroamericana y violencia de género: pesadilla sin límites. Revista del Centro de Estudios Interdisciplinario sobre Mujeres, 21(22), 75-86.

Bastia, T. \& Busse, E. (2011). Transnational Migration and Changing Gender Relations in Peruvian and Bolivian Cities. Diversities, 1(13), 19-34.

Bettio, F. et al. (2006). "Change in care regimes and female migration, the "care drain' in the Mediterranean". Journal of European Social Policy, 16(3), 271-285.

Castelló, L. (2009). La mercantilización y mundialización del trabajo reproductivo. El caso español. Revista de Economía Crítica, 7, 74-94.

Ciurlo, A. (2015). La migración femenina y los cambios en las relaciones de género en las familias: el caso de las transmigrantes colombianas en Italia. OASIS, (21), 55-79.

Conferencia Internacional del Trabajo (2017). Informe IV. Migración laboral: nuevo contexto y desafios de confianza. Ginebra, Suiza: Oficina Internacional del Trabajo.

Durán, M. A. (1972). El trabajo de la mujer en España. Un estudio sociológico. Madrid, España: Tecnos.

Ezquerra, S. (2010). La crisis de los cuidados: orígenes, falsas soluciones y posibles oportunidades. Viento Sur (108).

Ezquerra, S. (2012). Crisis de los cuidados y crisis sistémica: la reproducción como pilar de la economía llamada real. Investigaciones feministas, 2, 175-194.

Fuentes, V., \& Agrela, B. (2016). Migración femenina de "larga duración" y acuerdos de cuidados. Una mirada desde el Trabajo Social en origen y destino. Trabajo Social Global, 6(11), 31-53.

Gonzálvez, H. (2010). Migración Colombiana, género y parentesco: la organización social de los cuidados. Universidad de Granada, Departamento de Antropología Social, Programa de doctorado: Antropología y diversidad cultural, Tesis doctoral, Granada.

Hechavarría, M. M., Ramírez, M., García, H., 
\& García, A. (2018). El envejecimiento. Repercusión social e individual. Revista Información Científica, 97(6), 11731188.

Herrera, G., (2012). Género y migración internacional en la experiencia latinoamericana. De la visibilización del campo a una presencia selectiva. Política y Sociedad, 49, 1.

Hochschild, A. R. (2000). Global care chains and emotional surplus value. $\mathrm{En} \mathrm{W}$. Hutton y A. Giddens (eds.), On the edge. Living with global capitalism (pp.130146). New York: Vintage.

International Labour Organization (2015). ILO Global estimates of migrant workers and migrant domestic workers: results and methodology / International Labour Office. Turin, Italy: International Training Centre of the ILO.

Juliano, D. (2000). Mujeres estructuralmente viajeras: estereotipos y estrategias. Entrevista con Dolores Juliano. Papers $6 o, 381-389$.

Larrañaga, I., Arregui, B., \& Arpal, J. (2004). El trabajo reproductivo o doméstico. $G a-$ ceta Sanitaria, 18(Supl 1), 31-37.

Legarreta, M. (2017). Notas sobre la crisis de cuidados: distribución social, moralización del tiempo y reciprocidad del tiempo donado en el ámbito doméstico-familiar. Arbor, 193(784).

Martínez, R. (2010). Servicio doméstico y trabajo de cuidados: hacia la privatización del cuidado familiar. Alternativas, 17, 157-179.

Maruani, M. (ed.) (2000). Las nuevas fronteras de la desigualdad. Hombres y mujeres en el mercado de trabajo. Barcelona, España: Icaria.
Molano, A., Robert, E., \& García, M. (2012). Cadenas globales de cuidados: síntesis de resultados de nueve estudios en América Latina y España. Santo Domingo, República Dominicana: ONU Mujeres.

Monreal, M., Cárdenas, R., \& Martínez, B. (2019). Estereotipos, roles de género y cadena de cuidados. Transformaciones en el proceso migratorio de las mujeres. Collectivus, Revista de Ciencias Sociales, 6(1), 83-99.

Montoro-Gurich, C. (2017). Marroquíes en España: un análisis por género de los determinantes en las migraciones familiares. Estudios Geográficos, 78(283), 445-464.

Naciones Unidas. (2019). Naciones Unidas. Población. Recuperado de https://www. un.org/es/sections/issues-depth/population/index.html

OIM (2006). Derecho Internacional sobre Migración. Glosario sobre Migración. Ginebra, Suiza: Organización Internacional para las Migraciones.

O'Neil, T., Fleury, A., \& Foresti, M. (2016). Women on the move. Overseas Development Institute Briefing.

Organización Internacional del Trabajo (2018a). The women at work initiative. Geneva, Suiza: ILO.

Organización Internacional del Trabajo (2018b). ILO Global Estimates on International Migrant Workers. Results and Methodology. Geneva, Suiza: ILO.

Orozco, A. (2007). Cadenas globales de cuidado. Santo Domingo, República Dominicana: INSTRAW.

Oso, L., \& Parella, S. (2012). Inmigración, género y mercado de trabajo: una pano- 
rámica de la investigación sobre la inserción laboral de las mujeres inmigrantes en España. Cuadernos de Relaciones Laborales, (1), 11-45.

Parella, S. (2003). Mujer inmigrante y trabajadora. La triple discriminación. Barcelona, España: Anthropos.

Parella, S. (2003). Mujer, inmigrante y trabajadora, la triple discriminación. Barcelona, España: Anthropos.

Parella, S., \& Speroni, T. (2018). Las perspectivas transnacionales para el análisis de la protección social en contextos migratorios. Autoctonía. Revista de Ciencias Sociales e Historia, 2(1), 37-56.

Pedone C., Agrela, B. \& Gil, S. (2012). Políticas públicas, migración y familia. Una mirada desde el género. Papers, 3(97), 541-568

Pérez, A., Paiewonsky, D., \& García, M. (2008). Cruzando fronteras II: Migración y desarrollo desde una perspectiva de género. Madrid, España: Instituto de la Mujer y UN-INSTRAW.

Petit, J. M. (2002). Migraciones, vulnerabilidad y políticas públicas. Santiago de Chile, Chile: Conferencia hemisférica sobre migración internacional.
Rodríguez, A. \& Larrañaga, M. (2002-2004). El trabajo de las mujeres: claves para entender la desigualdad laboral. Universidad del País Vasco-Euskal Herriko Unibertsitatea.

Sassen, S. (1999). La ciudad global. Buenos Aires, Argentina: Universidad de Buenos Aires.

Solé, C. (2000). La inmigración femenina en la era de la globalización. En Mujer y migración en el mediterráneo occidental (pp. 241-253). España: Editorial Icaria.

Trujillo, M., \& Almeda, E. (2017). Monomarentalidad e imaginarios de género en contexto migratorio: Punto de vista epistemológico feminista en el estudio de las migraciones. EMPIRIA. Revista de $\mathrm{Me}$ todología de las Ciencias Sociales, (37), 101-125.

\section{PARA CITAR ESTE ARTÍCULO}

Cárdenas Rodríguez, R., y Ortega de Mora, F. (2019). Transmisión del trabajo reproductivo familiar hacia las mujeres migrantes. Vulnerabilidad, violencia e invisibilidad dentro del trabajo doméstico. Collectivus, Revista de Ciencias Sociales, 6(2), 105-119.

DOI: http//dx.doi.org/10.15648/Coll.2.2019.7

Recibido: 25/11/2018 Aprobado: 27/02/2019 